\title{
Genetic Optimization and Simulation of a Piezoelectric Pipe-Crawling Inspection Robot
}

\author{
Geoffrey A. Hollinger \\ Engineering Department \\ Swarthmore College \\ Swarthmore, PA 19081 United States \\ Geoff.Hollinger@gmail.com
}

\author{
Jeri M. Briscoe \\ Engineering Directorate 12 \\ NASA Marshall Space Flight Center \\ Huntsville, AL 35812 United States \\ Jeri.M.Briscoe@nasa.gov
}

\begin{abstract}
Using the Darwin2k development software, a genetic algorithm (GA) was used to design and optimize a pipecrawling robot for parameters such as mass, power consumption, and joint extension to further the research of the Miniature Inspection Systems Technology (MIST) team. In an attempt to improve on existing designs, a new robot was developed, the piezo robot. The final proposed design uses piezoelectric expansion actuators to move the robot with a 'chimneying' method employed by mountain climbers and greatly improves on previous designs in load bearing ability, pipe traversing specifications, and field usability. This research shows the advantages of $\mathrm{GA}$ assisted design in the field of robotics.
\end{abstract}

Index Terms - genetic algorithms, evolutionary robotics, inspection robots

\section{INTRODUCTION}

One of the major areas of research in robotic design is testing and modification of prototypes. Current robots have become so complex and difficult to manufacture that field-testing of many designs is economically unfeasible. Computer simulation offers an effective alternative with many advantages over actual fabrication of prototype designs.

One advantage of computer simulation is the ability to evaluate many designs quickly. Often a computer simulated run can take a few minutes where a real world run could take hours. One technique for taking advantage of this aspect of computer simulation involves a GA. If a robot is described as a bit string, a GA can modify this bit string to change the design of the robot based on a fitness function. For instance, you could optimize a robot for low mass or low power consumption by using a GA to modify the robots parameters and then simulating each configuration.

Using a GA for robot optimization has several notable advantages that stem from the nature of GA problem solving. For instance, GA assisted robot design is resistant to local minima. Unlike gradient descent methods for optimization, a GA will successfully sample out locally good fits for globally better fits. This is a great advantage in robotics because there are often many designs capable of performing the desired task. As an example, a GA, if allowed, will not hesitate to try a six-legged design even if a four-legged design is successfully completing the task. Gradient descent methods, on the other hand, will likely try to further optimize the four-legged design [1].
A GA can also solve many problems faster than random search methods. To design a robot, all configurations could be searched to determine the best fitness. With a GA, this is not necessary. Instead, poor configurations can be quickly sampled out of the population without leading to further evaluation of similar configurations. This allows for a quick computer design using current processing power.

A GA is also a powerful tool for optimizing prototype designs. Often during the design process, a prototype is developed that can complete the task but does not match the required specs for a final design. By using the prototype to generate an initial population and then allowing the GA to optimize for the necessary specs, it is possible to quickly move from the prototype design to the final design [2].

One program that takes advantage of a GA for evolutionary design is the Darwin $2 \mathrm{k}$ robot development package. Darwin2k was written by Dr. Chris Leger to allow for automated robot synthesis and design using embedded GA technology [3]. It contains built in robot components, controllers, evaluators, and 3D simulation code as well as some working demos from Leger's research. The program fully integrates a GA based population manager and provides a parametric representation of robot components to facilitate this design. Darwin2k uses 3D polyhedral terrain elements allowing for simulation of robots on various terrains specified by the user. The program is open source and written in $\mathrm{C}++$ allowing for modular modification and easy revision. The software is available along with many papers on evolutionary robotics at www.darwin $2 \mathrm{k}$.com.

Darwin2k describes robots as a list of linked components. For instance, a robot might consist of a base link, followed by arm links, hinge joints, and finally locomotion elements for interacting with the terrain. Each of these links has several parameters, such as length, width, range of extension, and position of connectors. The GA is allowed to change these parameters within ranges specified by the user. Fig. 1 shows the example of the specification of one such parameter. The first value, the const flag, tells the GA whether or not is can modify this parameter. The second and third values give the minimum and maximum that the GA can set this parameter. The fourth value tells the GA how many values between the minimum and maximum that it may use (denoted as $2^{\mathrm{n}}$ values). Finally, the last value is the starting value for that parameter. This allows the GA to view the changeable parameters as a bit string and modify values accordingly. 


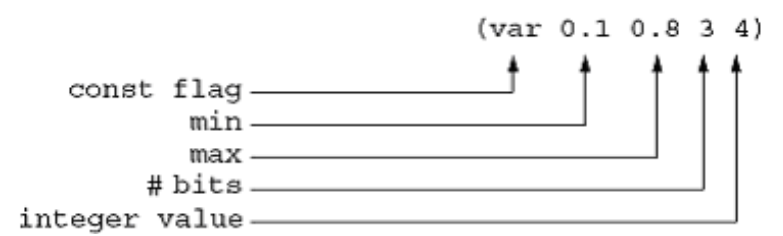

Fig. 1 Example of Darwin2k parameter description

Darwin2k's GA allows the user to modify mutation and crossover rates as well as population size and number of generations. It also contains more advanced GA features that were not used in this research [3]. After GA specification, Darwin2k performs fitness evaluation using user specified metrics. Some built in metrics include mass, power consumption, link deflection, and structural stability. After deciding on metrics, the user specifies how the metrics are used to determine fitness. Two possible choices are weighted average and prioritization. Using prioritization, the user can force robots to meet one metric before other metrics are even considered. This method becomes useful when evaluating robots for task completion. Often, if a robot cannot complete a desired task, other metrics such as power consumption do not matter.

Another excellent feature of Darwin2k's population manager is its ability to stochastically determine the initial population from a fully specified configuration. The user specifies a base configuration, such as a prototype design, and the GA generates the initial population by using statistical methods to span the entire design space. Since the prototype is included in the initial population, the GA already has a user specified design to beat. This takes advantage of both the user and the GA to influence the design process.

\section{PROBLEM DESCRIPTION}

The goal of the Miniature Inspection Systems Technology (MIST) team is to design and manufacture a robot capable of inspecting and removing debris from a $3 / 8$ ' inner diameter coolant pipe on the space shuttle main engine. To begin moving towards this goal, the MIST team has used GA assisted design to simulate a piezoelectric robot capable of traversing a bent pipe with a 1 " inner diameter and a $180^{\circ}$ bend radius of 4 "'.

When designing this robot, the goal was set that it should pull a load of at least two pounds. This corresponds to the approximate weight of the tethered wire needed to supply the robot with power and feed the vision data back to a host computer. Also, the goal was set that the robot should function in both the Earth's gravity and in microgravity. This is necessary since the robot must operate both on Earth and in orbit. For ease of use, the robot must move at a speed reasonable to semi-autonomous pipe inspection. Other goals included low mass and power consumption. Finally, since piezoelectric technology was used, consideration must be given to the manufacturability of the piezoelectric actuators.

\section{UMBRELLA ROBOT DESCRIPTION}

The starting point for the new robot was the umbrella robot designed by Mike Craft at Dynamic Concepts, Inc with Marshall Space Flight Center [4]. This robot uses 'chimneying' movement where it has front and back clamping joints connected by a prismatic element. A standard movement sequence goes as follows: the front clamper releases, the center element expands, the front clamper clamps, the back clamper releases, the center elements contracts, the back clamper clamps, the front clamper releases, the center element extends. Through this motion, the robot moves forward through the pipe.

While the 'chimneying' method of locomotion was retained in the final design, the umbrella robot had some major shortcomings that did not meet our project goals. The major issue with the umbrella robot was its thin, fragile components that did not hold up to the forces necessary to pull the two pounds of wire. Attempts to attach loads to the umbrella robot in simulation failed and deflected the robot members beyond structural integrity limitations. The small pipe clamping feet were also problematic because they did not provide sufficient contact surfaces (approximately 1 $\mathrm{mm}^{2}$ ) to avoid slipping. Furthermore, the design did not specify the nature of the motors and forces necessary to drive the robot. It would be difficult to manufacture moving parts at such a small scale, and the motors would not produce enough force to pull the load or supply the normal force for frictional pipe contact. Despite these shortcomings, the umbrella robot was a useful starting point, and much was gained from the controller and configuration design. Fig. 2 displays a picture of the umbrella robot simulated in a bent pipe.

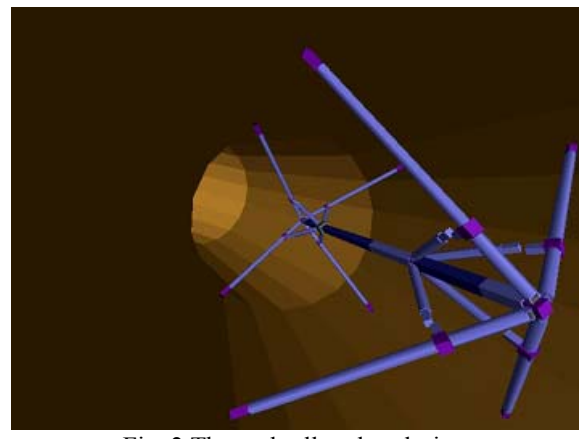

Fig. 2 The umbrella robot design

\section{GA OPTIMIZATION OF UMBRELLA ROBOT IN GRAVITY}

Another shortcoming of the umbrella robot design was its inability to function in Earth's gravity. To test the functionality of the Darwin2k GA, adaptive optimization of the umbrella robot in gravity was performed. The simulated acceleration due to gravity was increased from $0.5 \mathrm{~m} / \mathrm{s}^{2}$ (the upper functional limit for the initial umbrella robot) to 1.5 $\mathrm{m} / \mathrm{s}^{2}$. The population manager was then set up to judge the robot on task completion, mass, power consumption, link deflection, and time. Top priority was given to task completion because umbrella robots unable to move through the pipe were useless. The task was specified as moving through two meters of straight pipe. The GA was allowed 
to modify the length of the center prismatic element and the lengths of the umbrella robot's legs. Table I gives the GA parameters used for this optimization and the run time on a Pentium IV $2.8 \mathrm{GHz}$ processor.

TABLE I

$\begin{array}{cc}\text { GA PARAMETERS FOR UMBRELLA ROBOT OPTIMIZATION IN GRAVITY } \\ \text { Population Size } & 10 \\ \text { Crossover Rate } & 0.95 \\ \text { Mutation Rate } & 0.05 \\ \text { \# of Generations } & 10 \\ \text { Run Time (hrs) } & 1.5\end{array}$

Through the use of the GA, the upper functional limit of the umbrella robot was increased to $1.5 \mathrm{~m} / \mathrm{s}^{2}$. The GA's modification of the robot's leg and center joint dimensions resulted in changes in leverage for the back and front clampers. As a result, the robot could withstand higher gravitational pull. Table II gives the initial and optimized values. This shows a successful run of the Darwin $2 \mathrm{k}$ population manager and embedded GA design. Through this process, however, we were unable to achieve functionality of the umbrella robot in gravity close to that of the Earth. As a result, as well as considering the other design issues described above, the umbrella robot design was thrown out.

TABLE II

$\begin{array}{lll}\text { CHARACTERISTICS OF INITIAL AND OPTIMAL UMBRELLA ROBOT } & \text { Optimal } \\ & \text { Initial } & \text { Optic } \\ \text { Center prismatic } & 13.5 \mathrm{~mm} & 13.75 \mathrm{~mm} \\ \text { Front legs } & 14 \mathrm{~mm} & 13.8 \mathrm{~mm} \\ \text { Back legs } & 13 \mathrm{~mm} & 13.3 \mathrm{~mm} \\ \text { Gravity limit } & 0.5 \mathrm{~m} / \mathrm{s}^{2} & 1.5 \mathrm{~m} / \mathrm{s}^{2}\end{array}$

\section{Designing A Simulated Curved PiPe}

To meet the problem specifications, a new pipe needed to be rendered to model the $180^{\circ}$ turn and exact dimensions in Darwin2k. Previous obstacles, such as those used by the umbrella robot, were described by hand using user specified coordinates and vertex plotting. Previous pipes only consisted of five polygons and did not show any curvature [4]. Since a higher polygon resolution and exact curvature specifications were desired, a program was written to convert an ASCII scene description (.ASE) to a Darwin2k terrain obstacle. The program also specifies the coefficients of dynamic and static friction ( 0.47 and 0.61 respectfully for aluminum on steel) necessary for terrain specification. This allows for any ASCII scene file to be converted to a Darwin2k obstacle. 3D Studio Max was used to design a 432 polygon pipe scaled to 1 " inner diameter and 4 "' bend radius. This pipe is easily applicable to any robot configuration. Fig. 3 shows the pipe designed for this paper. Both 3D Studio Max and Maya will export into the ASCII scene description, and these files can now be imported into Darwin2k.

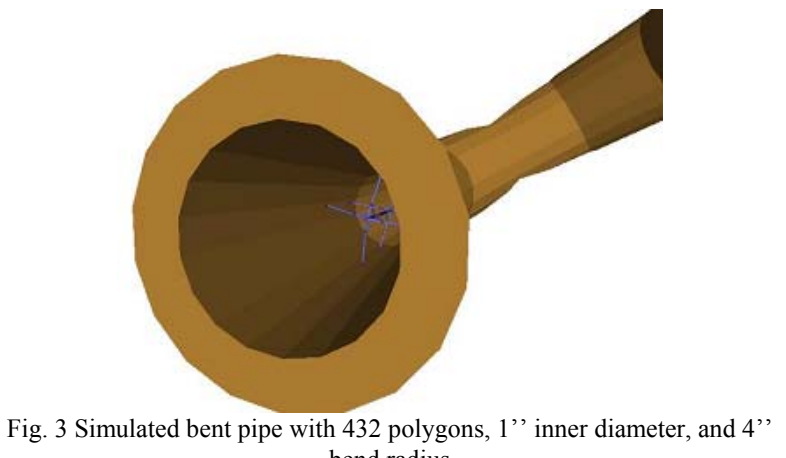

$$
\text { bend radius }
$$

\section{INITIAL PIEZo RoBot DESIGN}

To begin the design of the piezo robot, many considerations were taken into account. The initial design was constructed to fit the mechanical specifications from Dynamic Structures and Materials, LLC. It uses the same 'chimneying' movement concept as the umbrella robot. Due to limitations on current piezoelectric technology, the expansion of the front clampers was initially limited to 300 microns, and the expansion of the center prismatic element was limited to 500 microns. Also, the forces of extension of the joints were examined in Darwin2k to reach approximately those specified by piezoelectric actuators. Attention was also paid to the dimensions and shape of the robot components requiring extensive additions to the Darwin2k robot component library. The front and back clamper design did not exist in the software, and the terraininteracting feet needed to by modified. The contact surface with the pipe also needed to be examined and fit the $28 \mathrm{~mm}^{2}$ specification. This is 28 times the contact surface of the umbrella robot. Also, the controller needed to be examined and fit to run on a power input similar to that of piezoelectric motors. The force of the center joint and load bearing ability was also taken into consideration as well as the mass of the robot. To make the robot as light as possible, all metal components were simulated as aluminum at this stage in the design. Fig. 4 shows a picture of a manufactured prototype of the piezoelectric robot. Fig. 5 shows a drawing of the prototype, and Fig. 6 shows the simulated design of the initial robot.

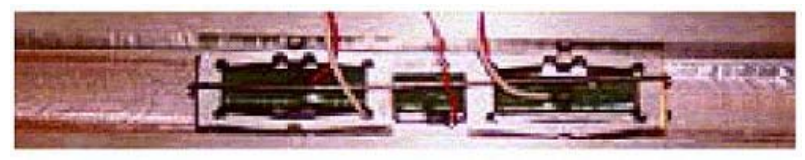

Fig. 4 Picture of manufactured prototype piezo robot

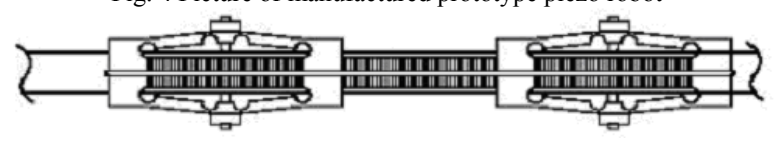

Fig. 5 Drawing of piezo robot

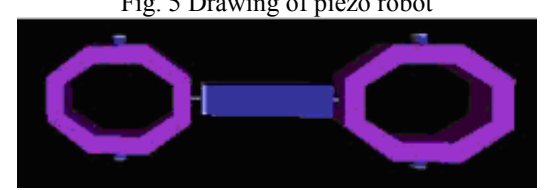

Fig. 6 Simulated piezo robot prototype 


\section{Piezoelectric ExPANSION ACTUATORS}

To attain a realistic simulation of the piezo robot, research was done on the manufacture and control of piezoelectric actuators. Due to the inverse piezoelectric effect, some crystal materials will exhibit strain when faced with an applied electrical field. Constrained by the piezoelectric modulus of the material, these materials will expand relative to an applied voltage. Materials that exhibit this property include lead-zirconate-titanate (PZT), leadtitanate $\left(\mathrm{PbTiO}_{2}\right)$, lead-zirconate $\left(\mathrm{PbZrO}_{3}\right)$, and bariumtitanate $\left(\mathrm{BaTiO}_{3}\right)$. For correct expansion, piezoelectric materials must be formed from single crystals and constrained properly. Once manufactured, they can only be driven at certain resonant frequencies [5]. The piezoelectric actuators manufactured for the piezo robot will be made out of PZT, so these properties were used to determine the forces and power consumption of the simulated robot. A more realistic piezoelectric expansion model is suggested later. Fig. 7 shows pictures of manufactured piezoelectric actuators and a picture of a simulated actuator.
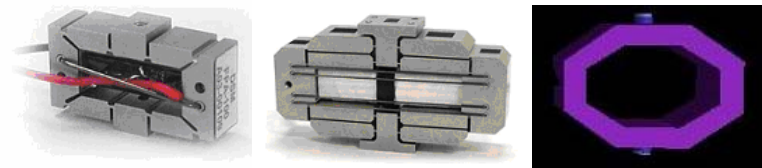

Fig. 7 Manufactured actuators (left and center) and simulated actuator (right)

Piezoelectric actuators have the advantage of precise extension and movement. Also, they do not contain motors, gears, or other moving parts that have a high likelihood of failure. The most important property of piezoelectric actuators for this design is their fabrication on the microscale level. Some disadvantages of using piezoelectric actuators are their high power consumption and the difficulty to improve extension beyond mechanical limits.

To control a robot using piezoelectric actuators, a controller with fairly high power consumption must be used. The controller being designed for the piezo robot inputs a $120 \mathrm{~V} \mathrm{p} / \mathrm{p}$ non-sinusoidal wave driving each of the clampers and the center joint. The controller uses $1.5 \mathrm{~kW}$ of power and operates at a frequency of $1400 \mathrm{~Hz}$. Fig. 8 shows a sample controller input to a piezoelectric robot. Unfortunately, Darwin2k did not allow for implementation of this specific controller. Instead, the piezo robot was designed to run on similar power with similar forces of extension. A more realistic controller design is suggested later.

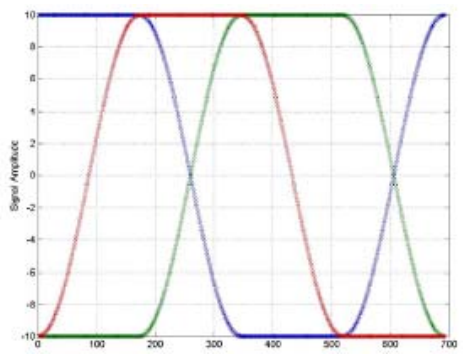

Fig. 8 Sample wave input for front and back clampers (blue and green) and center joint (red)

\section{SUGGeSTED PIEZO RoBot IMPROVEMENTS FROM SiMULATION}

The first problems encountered when simulating the piezo robot was failure to navigate the 4" turn radius. Many configurations could not to negotiate tight turns or pipe deformations without becoming lodged in the pipe. In attempt to fix this, an elastic hinge joint was implemented. This joint provided a rotational correcting moment to the bent hinges, based on a spring constant, in hopes of allowing it to correct itself after bending. The hinge joint failed to resolve the problem, and the elastic hinged robot still crashed in high distortion areas of the pipe. Fig. 9 shows a crash due to hinge joint failure.

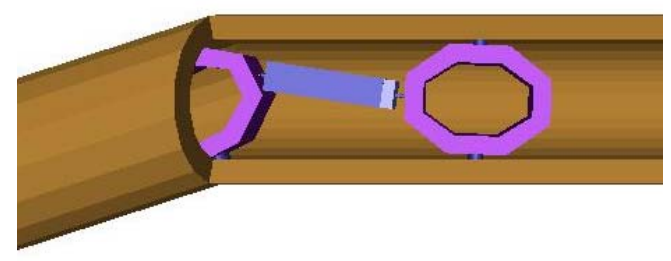

Fig. 9 Piezo robot crash due to hinge joint failure

To resolve the crashing problems, the expansion of the center prismatic joint was increased beyond manufacturable specifications. This greatly increased the maneuverability and speed of the robot and allowed it to negotiate extreme pipe deformation. While this solution presents concerns for real world application, expansion of piezoelectric material is constantly improving. Also, real world robots should not see the extreme pipe deformations presented as obstacles in simulation.

Once the crashing issues were resolved, robots smaller than manufacturable limits were simulated. These robots performed favorably at task completion. This shows that further miniaturization and hopes of reaching the $3 / 8$ ' inner radius are conceptually possible using piezoelectric actuators

\section{GA Optimization of Piezo Robot Design}

After the initial design and simulation of the piezo robot was finished, the GA was used to optimize several parameters. The first task was to add link extension to the parameters that the GA could optimize. The hope was that the extension of the center and clamper joints could be reduced to decrease manufacturing difficulty.

After joint extension was added to the GA, a configuration was setup to allow the GA to modify the dimensions, extension, and material of all components (a total of 15 parameters in all). Fitness was evaluated with a weighted average of the following parameters: task completion, low power consumption, small mass, and short joint extension. Unfortunately, a weighting function could not be developed that was sufficient to allow for all of these parameters to be optimized simultaneously. A complete optimization strategy is suggested later. The designated task was for the robot to successfully navigate the first curve in the pipe. 
To successfully optimize the robot, separate GA runs were done with each giving priority to a separate metric. The separate optimizations produced three feasible designs with different optimized parameters. Depending on the application, any of these designs can be chosen. The same GA specification was used for these runs as for the umbrella robot (see Table I). The GA took approximately 2.5 hours to run on the same P IV $2.8 \mathrm{GHz}$ computer. Table III shows the statistics of the initial and optimized robots.

These results show that the GA runs were successful in reducing the power consumption, mass, and link extension of their respective configurations. The GA increased the frictional contact area and shortened the length of the actuators to minimize joint extension. It decreased the mass by making the front and back actuators thinner. Finally, it decreased the power by decreasing the mass and decreasing the extension of the front and back actuators. Each of these designs has unique strengths and weaknesses and can be chosen for specific tasks.

TABLE III

SPECIFICATIONS OF OPTIMIZED CONFIGURATIONS Initial Design Extension Optimal Mass Opt. Power Opt.

$\begin{array}{lcccc}\begin{array}{l}\text { Clamper } \\ \text { Extension } \\ \text { Center }\end{array} & 200 \mu \mathrm{m} & 475 \mu \mathrm{m} & 300 \mu \mathrm{m} & 200 \mu \mathrm{m} \\ \text { Extension } & 3.00 \mathrm{~mm} & 2.75 \mathrm{~mm} & 4.75 \mathrm{~mm} & 5.00 \mathrm{~mm} \\ \text { Mass } & 20.8 \mathrm{~g} & 20.1 \mathrm{~g} & 13.9 \mathrm{~g} & 15.2 \mathrm{~g} \\ \text { Power } & 1514 \mathrm{~W} & 982 \mathrm{~W} & 996 \mathrm{~W} & 584 \mathrm{~W}\end{array}$

The major concerns from the simulation results stem from the large extension of the center joint. Due to distortions inherent in polygonal simulation of the pipe terrain, the simulated robot's center extension joint needed to extend well beyond manufacturable limits. A higher polygon resolution pipe would have been desirable, but runtime constraints on the GA limited the complexity of the pipe. To address this issue, a robot with manufacturable piezoelectric actuators was simulated for each of the optimal robot configurations and found to be capable of traversing a more realistic pipe of higher polygon resolution. The final manufacturable robot dimensions are given in Appendix B.

\section{CONCLUSIONS}

Through simulation, the piezo robot is now capable of traversing a simulated 1 ', inner diameter pipe with a $180^{\circ}$ turn and 4" bend radius. The piezoelectric actuators allow for high force movement. They can easily pull the specified $2 \mathrm{lb}$ load and operate both in the Earth's gravity and in microgravity. Figures 10-11 show snapshots of the robot performing these tasks.

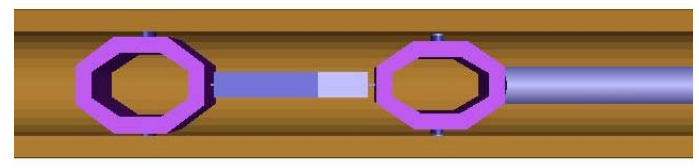

Fig. 10 Piezo robot carrying $2 \mathrm{lb}$ load

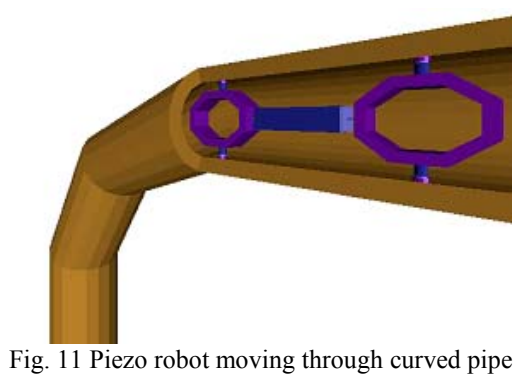

Through the use of Darwin2k's GA, piezo robot configurations are now available with power usage down to 584 watts and robot mass down to 13.9 grams. These low values show the success of the GA at optimizing single performance metrics while still maintaining task completion. Even the final designs, however, consume considerable power and are not resilient to distortions in the pipe. From simulation, it has been shown that further miniaturization is possible, but techniques must be developed to bypass distortions and pipe debris.

Evaluating Darwin2k, the software proved excellent for $3 \mathrm{D}$ robotic simulation and design. The population manager had great options and capability to evolve robots and implement the embedded GA. The robot component library, however, proved to be fairly limited, and many components needed to be added or redesigned for the piezo robot. Furthermore, Darwin2k was difficult to use and would greatly benefit from a GUI. The open source nature of the software provided advantages in that it could be modified, but a user without knowledge of $\mathrm{C}++$ would not be able to do this.

\section{Future RESEARCH}

For future work, Darwin2k should be modified to allow optimization of more parameters. For instance, modification of controller gains would allow the GA to reduce power consumption and increase speed. Also, the metric weighting functions should be examined and more research done to allow for optimization of many parameters instead of the separate optimization of each. The piezo robot would also benefit from a more realistic model of the piezoelectric expansion actuators and their controller. Some of the actual piezoelectric equations should be programmed into Darwin $2 \mathrm{k}$, and the controller input should be similar to that described in the previous section. A GUI or Windows port for Darwin2k to address the ease of use issues should also be examined.

In addition, a more realistic model of the pipe terrain should be explored. If parallel processing were used, a higher polygon resolution pipe could be implemented while maintaining reasonable GA runtimes. This would lessen the unrealistic distortions in the pipe that were forcing center joint extensions beyond manufacturable limits in the simulated robots. Additionally, a more complex modeling of the contact forces between the robot and terrain would help to improve the realism of the simulation.

To further GA use in robotic design, evolutionary capabilities should be explored beyond simple optimization. Darwin2k allows for limited evolution of complete robots to perform specified tasks. The piezo robot did not use this 
technique, but further modifications to Darwin $2 \mathrm{k}$ could allow for pipe crawlers to be designed entirely using GA's. The GA could decide which components to place on the robot and how many of such components to use. For a robot as complex as the piezo robot, this would require a huge amount of processing power. If the processing power were available, such research could lead to field evolvable robots that adapt to their surroundings and fix failures automatically [6].

Further tasks for the MIST team include simulation of the camera and debris removal mechanisms on the piezo robot. After these designs have been confirmed, the robot should then be manufactured, assembled, and tested in a real pipe. After this has been done, MIST can move on to design robots for other applications using similar techniques as the one used here.

\section{ACKNOWLEDGMENT}

Thanks to Undergraduate Student Research program coordinator Julie Mills at NASA Marshall Space Flight Center. Further thanks to Ken King and Jason Waggoner in Engineering Directorate 12 at Marshall. Thanks to Jeff Payne at Dynamic Structures and Materials, LLC for mechanical consultation and to Mike Craft at Dynamic Concepts, Inc for use of the umbrella robot design.

\section{REFERENCES}

[1] Leger, Chris and J. Bares, "Automated Synthesis and Optimization of Robot Configurations". Proceedings of the 1998 ASME Design Engineering Technical Conferences, Atlanta, GA.

[2] Leger, Chris. "Performance Characterization of an Automated System for Robot Configuration Synthesis". In Proceedings of SPIE Vol. 4196, November 2000.

[3] Leger, Chris. "Darwin2K: An Evolutionary Approach to Automated Design for Robotics," Kluwer Academic Publishers, 2000.

[4] Mike Craft and Tom Howsman, Dynamic Concepts, Inc. Dan O'Neil and Jim Steincamp, NASA Marshall Space Flight Center.

"Evolutionary Design and Simulation of a Tube Crawling Inspection Robot," Huntsville Simulation Conference, 2002.
[5] Cook, Gordon. "An Introduction to Piezoelectric Motors," Sensor Technology and Design, December 2001.

[6] Bongard, Josh C. and Hod Lipson. "Automated Robot Function Recovery after Unanticipated Failure or Environmental Change using a Minimum of Hardware Trials," Proceedings of the NASA/DoD Conference on Evolution Hardware, 2004.

\section{APPENDIX A: SOURCE CODE AdDITIONS TO DARWIN2K}

This section has been intentionally left out. Please email Geoffrey A. Hollinger (Geoff.Hollinger@gmail.com) for source code.

\section{APPENDIX B: DimENSIONS OF MANUFACTURABLE DESIGNS}

This section contains the dimensions of each of the manufacturable piezo robot designs. It should be noted that the center joint extension was decreased from the GA suggested values to meet realistic design constraints (see Section IX).

TABLE IV

RoBOt DIMENSIONS FOR OPTIMAL DESIGNS

Initial Mass Power

Design Optimized Optimized

Center Extension

(modified)

$500 \mu \mathrm{m} \quad 500 \mu \mathrm{m} \quad 500 \mu \mathrm{m}$

Center Beam Length $24.0 \mathrm{~mm} \quad 20.5 \mathrm{~mm} \quad 25.5 \mathrm{~mm}$

Center Beam Width $\quad 6.0 \mathrm{~mm} \quad 5.25 \mathrm{~mm} \quad 5.5 \mathrm{~mm}$

Center Beam Material Aluminum Aluminum Steel

Clamper Major

Radius

Clamper Minor

Radius

$9.5 \mathrm{~mm}$

$11.5 \mathrm{~mm}$

$9.5 \mathrm{~mm}$

Clamper Width

Clamper Shell

Thickness

$\begin{array}{ll}7.5 \mathrm{~mm} & 7.5 \mathrm{~mm} \quad 7.5 \mathrm{~mm}\end{array}$

$6.5 \mathrm{~mm}$

$5.0 \mathrm{~mm}$

$5.5 \mathrm{~mm}$

Clamper Material

$2.5 \mathrm{~mm}$

$2.0 \mathrm{~mm}$

$3.25 \mathrm{~mm}$

Clamper Extension

Aluminum

Aluminum

$200 \mu \mathrm{m}$

$300 \mu \mathrm{m}$

$200 \mu \mathrm{m}$ 